\title{
Development and growth of the foot lumbricalis muscle: a histological study using human foetuses
}

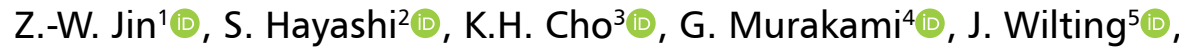 \\ J.F. Rodríguez-Vázquez ${ }^{6}$ (i)
}

${ }^{1}$ Department of Anatomy, Wuxi School of Medicine, Jiangnan University, Wuxi, Jiangsu, China ${ }^{2}$ Department of Anatomy, Division of Basic Medicine, Tokai University School of Medicine, Isehara, Japan ${ }^{3}$ Department of Neurology, Wonkwang University School of Medicine and Hospital, Institute of Wonkwang Medical Science, Iksan, Republic of Korea

${ }^{4}$ Division of Internal Medicine, Cupid Clinic, Iwamizawa, Japan

${ }^{5}$ Department of Anatomy, School of Medicine, Georg-August-Universität Göttingen, Germany

${ }^{6}$ Department of Anatomy and Embryology, School of Medicine, Complutense University, Madrid, Spain

[Received: 9 July 2020; Accepted: 12 August 2020; Early publication date: 2 September 2020]

Background: Our group has shown early development of the hand lumbricalis and hypothesized that, at midterm, the lumbricalis (LU) bundles flexor tendons to provide a configuration of "one tendon per one finger" (Cho K.H. Folia Morphol. 2012; 71, 3: 154-163). However, the study concentrated on the hand and contained no sections of near-term foetuses.

Materials and methods: The present examination of paraffin-embedded tangential sections along the planta from 25 embryos and foetuses at 6-40 weeks (15-320 mm crown-rump length) demonstrated that, at 8 weeks, the initial foot $L U$ appeared in the proximal side of the common tendinous plate of all five deep tendons.

Results: After midterm, a drastic three-phase change occurred at the muscle origin: 1) the LU originated from each of the flexor digitorum longus tendon (FDLT), but abundant tenocyte candidates separated the muscle fibre from the tendon collagen bundle; 2) the $L U$ arose from the covering fascia depending on increased thickness of the muscle; and 3) the LU muscle fibres intermingled with tendon collagen bundles and partly surrounded the tendon. Simultaneously, a dividing site of the FDLT migrated distally to accelerate the changes at the LU origin. These phases did not always correspond to the size of foetus after 30 weeks.

Conclusions: Consequently, in contrast to the hand $L U$, the delayed changes in the foot were characterised by involvement of the $L U$ origin into a single common part of the FDLT. The quadratus plantae muscle fibres did not attach to the $L U$ at any phase, and connected with the fourth and fifth toe tendons. (Folia Morphol 2021; 80, 4: 904-915)

Key words: flexor digitorum longus muscle, flexor halluces longus muscle, quadratus plantae muscle 


\section{INTRODUCTION}

The lumbricalis (LU) in the foot seems to be considered a small muscle, the function of which is limited because minute control is unnecessary at the phalangeal joints of the toe and because the muscle often shows variations, including absence $[17,18]$. Indeed, previous studies of finger tendons did not consider LU attachment [3, 8]. However, Cho et al. [6] paid special attention to early development of the hand LU and hypothesized that, at midterm, the LU rearranges the deep flexor tendons to provide a configuration of "one tendon per one finger". This hypothetical contribution of the LU onto tendon splitting from the common tendinous plate seems to be consistent with the fact that, in adults, crisscrossing of the deep tendon fibres frequently occurs at the LU muscle origin [13]. Although Cho et al. [6] tried to compare between the hand and foot, their study contained few observations of the foot and no demonstration of the near-term morphology. Near-term foetuses were likely to carry morphologies that were the same as or similar to that in children.

Dylevsky [8] reported, in contrast to superficial flexure tendons, that a single common tendon is transiently built by the union of tendons from the flexor digitorum profundus and flexor pollicis longus in human foetus hands. His reported "united tendon" was most likely to correspond to a "common tendinous plate" described by Cho et al. [6]. The common tendinous plate is seen much later than the finger separation with apoptosis. People may consider that a splitting of the common plate occurs by mechanical stress from the muscle movements of each finger. Further, the finger movement is likely to accelerate the tendon splitting. Therefore, how and when the common tendinous plate disappears is an interesting viewpoint in the human foetal anatomy. However, independent movement of a single finger (e.g., such as that of a pianist) seems to be unnecessary for the foetus foot. Cho et al. [6] failed to find the plate in the foot at midterm, possibly because almost all of their observations were based on transverse sections. Rather than transverse sections, sectional planes tangential to the hand palm or foot planta aspect provide a much better understanding of the anatomy of the LU and flexor tendons.

In contrast to the flexor digitorum profundus tendons in the hand palm, the flexor digitorum longus tendon (FDLT) is one tendon at the posterior half of the planta and divides into four tendons at the mid-planta near the metatarsal joints. However, there seemed to be no information regarding the topographical relation of the LU origin with the dividing site of the FDLT. We do not know whether the LU plays a role in the re-arrangement of FDLT at or after division. Likewise, morphology near term is also interesting because toe movements in utero may accelerate tendon division. Consequently, using tangential sections, the aim of this study was to clarify the foetal morphology of the LU muscle origins with special reference to the topographical relation to the four growing deep tendons.

\section{MATERIALS AND METHODS}

The study was performed in accordance with the provisions of the Declaration of Helsinki 1995 (as revised in 2013). We examined the paraffin-embedded histology of 28 embryos and foetuses at 6-15 weeks of estimated gestational age (crown-rump length [CRL] 15-118 mm): 7 embryos and early foetuses at $6-8$ weeks (CRL 15-31 mm), 6 foetuses at 12-15 weeks (CRL 70-118 mm), and 15 foetuses at 31-40 weeks (CRL 260-320 mm).

Five embryonic and three foetal specimens belonged to the Blechschmidt collection at the Medical Museum of Georg-August-Universität Göttingen. Although the sectional plane was sagittal to the head and trunk, we found five specimens containing a tangentially-cut foot. Most sections were stained with haematoxylin and eosin (HE), and a small amount were stained with azan or Masson trichrome. The use of this collection did not require specific approval of the Institute. The other 20 foetuses were a part of the large collection kept at the Department of Anatomy and Embryology, School of Medicine, Universidad Complutense, Madrid; the foetuses were the result of miscarriages and ectopic pregnancies at the Department of Obstetrics of the University. All sections were tangential along the plantar or palmar aspect and they were stained with HE. The use of the Spanish specimens was approved by the Complutense University Ethics Committee (B08/374).

\section{RESULTS}

\section{Observations of embryos and early and midterm foetuses}

At 6 weeks, the lumbricalis did not yet appear (Fig. 1). Rather than along the superficial side, the flexor digitorum brevis tendon lied between the FDLT: thus, the superficial and deep tendons appeared to be intercalated (Fig. 1A-C). A single FDLT ran distally along a long course in almost two-thirds of the planta. In the 


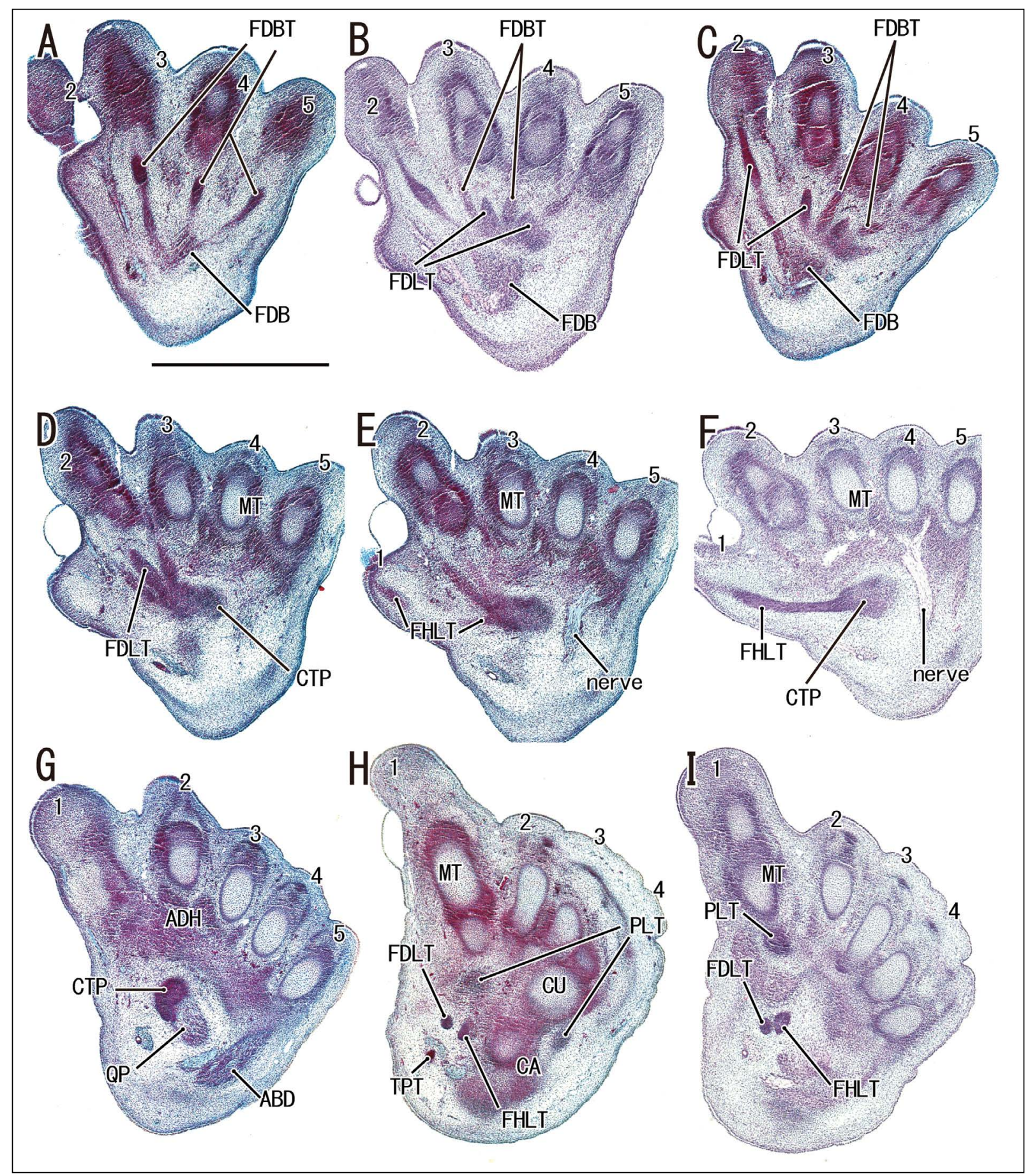

Figure 1. A-F. Early development of the flexor tendons in the foot planta. Crown-rump length $15 \mathrm{~mm}$. Tangential sections along the planta. Haematoxylin and eosin staining. Panel A displays the most superficial plane near the skin, while panel I exhibits the deepest plane in the figure. In panels A-C, the flexor digitorum brevis tendon (FDBT) appears to interdigitate with the flexor digitorum longus tendon (FDLT). In panels D-F, the flexor halluces longus tendon (FHLT) joins the FDLT to provide a common tendinous plate (CTP). The quadratus plantae (QP) inserts to the posterior margin of the common plate (panel G). All panels were prepared at the same magnification (scale bar in panel $A, 1 \mathrm{~mm}$ ). Either the lumbricalis or interosseous is not yet developed; $\mathrm{ABD}$ — abductor digiti minimi; $\mathrm{ADH}$ — adductor hallucis; $\mathrm{CA}$ — calcaneus; CU — cuboid; FDB — flexor digitorum brevis; MT — metatarsal bone; PLT — peroneus longus tendon; TPT — tibialis posterior tendon.

deep and distal side of the muscle belly of the flexor digitorum brevis, the FDLT joined the flexor halluces longus tendon to provide a common tendinous plate
(Fig. 1D-F). The quadratus plantae inserted to the deep and posterior part of the common plate (Fig. 1G). At 7-8 weeks, however, the flexor digitorum brevis 


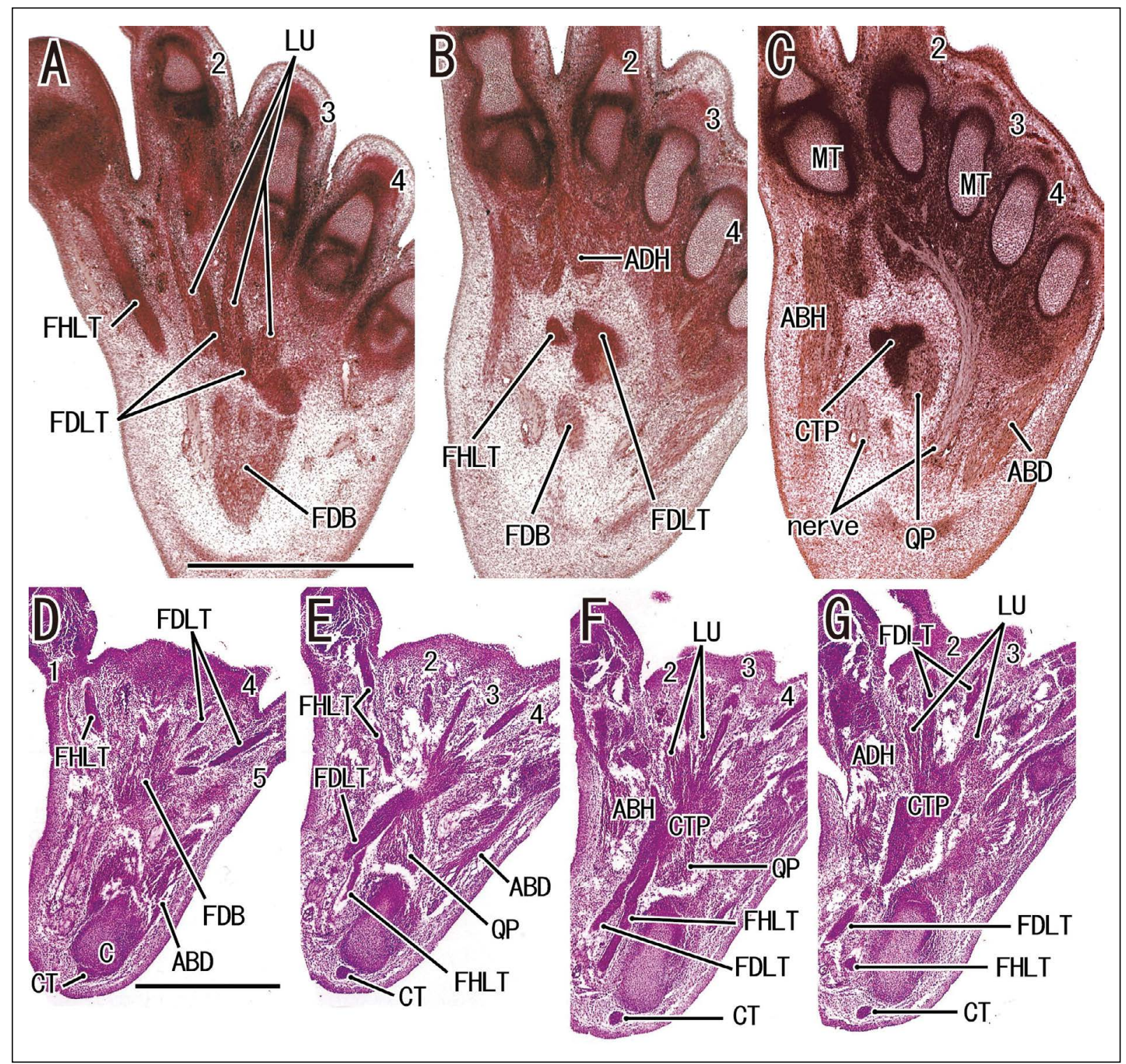

Figure 2. A-G. Early development of the foot lumbricalis; A-C. Crown-rump length (CRL) $30.5 \mathrm{~mm}$; D-G. CRL $28 \mathrm{~mm}$. Tangential sections along the planta. Haematoxylin and eosin staining. Panels $A$ and $D$ display the most superficial plane near the skin in each specimen. In panels $A$ and $F$, the initial lumbricalis (LU) is lying between the flexor digitorum longus tendons (FDLT, numbers indicate the corresponding finger). In panels B, C, F and G, the flexor halluces longus tendon (FHLT) joins the FDLT to provide a common tendinous plate (CTP). The quadratus plantae (QP) inserts to the common plate (panels $C, F$ ). Panels A-C or D-G were prepared at the same magnification (scale bars in panels $A$ and $\mathrm{D}, 1 \mathrm{~mm}$ ); $\mathrm{ABD}$ - abductor digiti minimi; $\mathrm{ABH}$ — abductor hallucis; $\mathrm{ADH}$ - adductor hallucis; $\mathrm{C}$ — calcaneus; $\mathrm{CT}$ - calcaneus tendon; FDB — flexor digitorum brevis; MT — metatarsal bone.

tendon was laid over and along the superficial aspect of the FDLT. The initial LU appeared between the FDLT (Fig. 2). Thus, it was located in the deep side of the superficial tendons. Notably, the common tendinous plate appeared not to attach to the initial lumbricalis, but it extended in a layer deeper than the LU (Fig. 2A, B). However, after enlargement of the common tendinous plate, the lumbricalis proximal end appeared to attach to the plate (Fig. 2F, G).

We failed to obtain complete tangential sections at midterm, but the sectional plane was much or less tilted (Fig. 3). Moreover, abortion manipulation might provide abundant red blood cells scattering in and between muscles. A common tendinous plate had already disappeared between the FDLT and flexor hallucis longus tendon. The foot lumbricalis did not originate from the common plate, but from the FDLT itself (Fig. 3D-F).

\section{Observations of near-term foetuses}

At the beginning of this study, we were confused regarding the variations in shape, location, and origin of the LU in near-term foetuses because multiple 


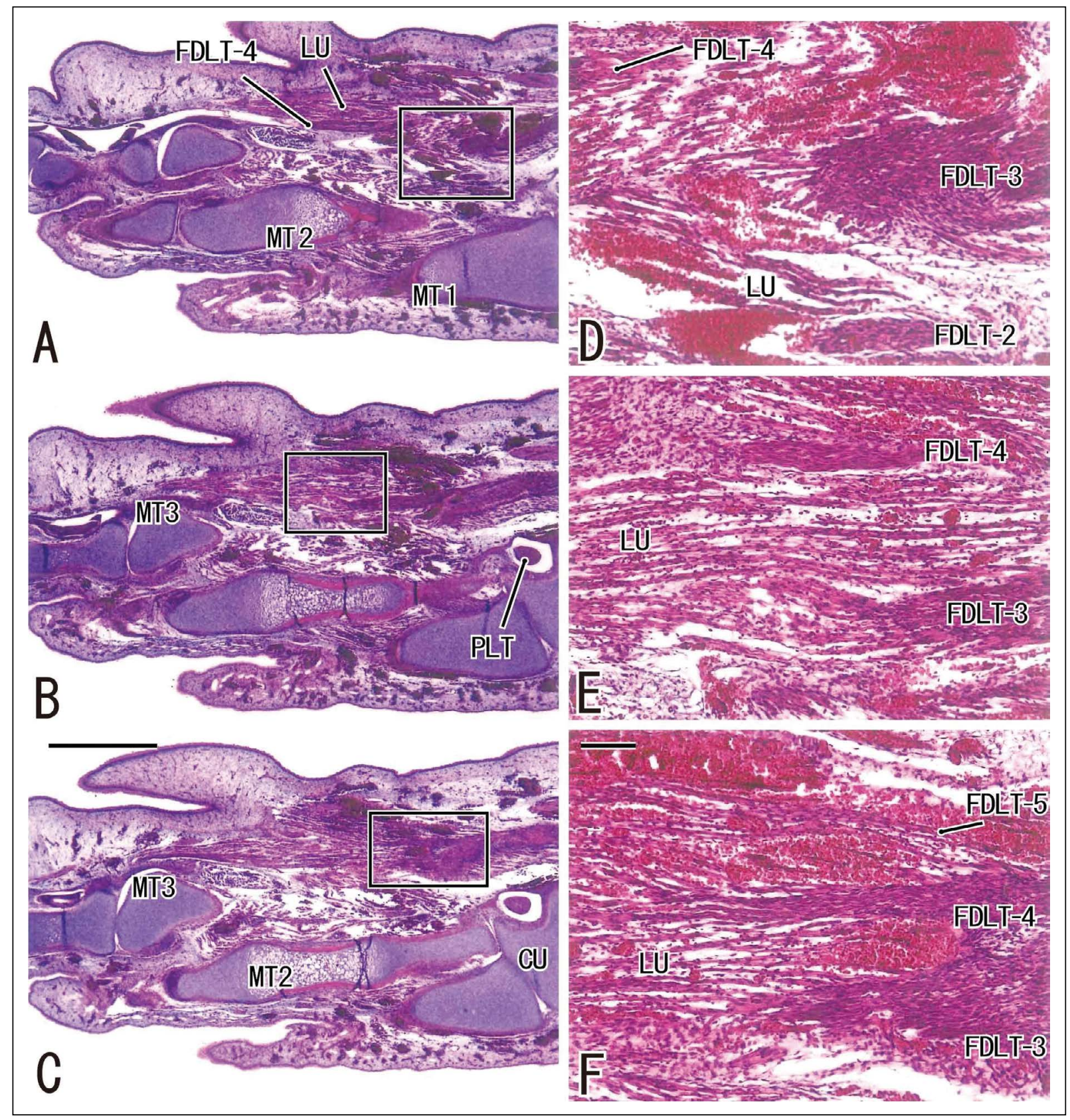

Figure 3. A-F. Foot lumbricalis at midterm. Crown-rump length $118 \mathrm{~mm}$. Tilted tangential sections along the planta. Haematoxylin and eosin staining. Panel A displays the most superficial plane near the skin in the figure. Panels D-F are higher-magnification views of squares in panels $A-C$, respectively. The lumbricalis (LU) originate from the flexor digitorum longus tendon (FDLT): the third toe tendon (panel $E$ ) and the third-fifth tendons (panel F). Abortion manipulation might result in abundant red blood cells that scatter in and between the muscles. Panels A-B (or D-F) were prepared at the same magnification (scale bar: $1 \mathrm{~mm}$ in panel A; $0.1 \mathrm{~mm}$ in panel F); CU — cuboid; MT — metatarsal bone; PLT — peroneus longus tendon.

stages appeared to be mixed in a single section. After 30 weeks, the LU morphology as well as the growth was different between specimens irrespective of the age or size. Below, we describe the most likely sequence of the morphological changes.

First, the LU muscle origin from the FDLT appeared to be established in the distal side of the dividing site of the tendon (Fig. 4A-F). The muscle fibre did not connect to the tendon collagen bundle; however, there was a narrow interface tissue containing abundant cells presumed tenocytes (Fig. 5A-C). Second, the LU grew to provide a thick muscle belly at the posterior or proximal half and was covered by fascia (Fig. 6A-C). Because of the thick muscle belly, the LU 

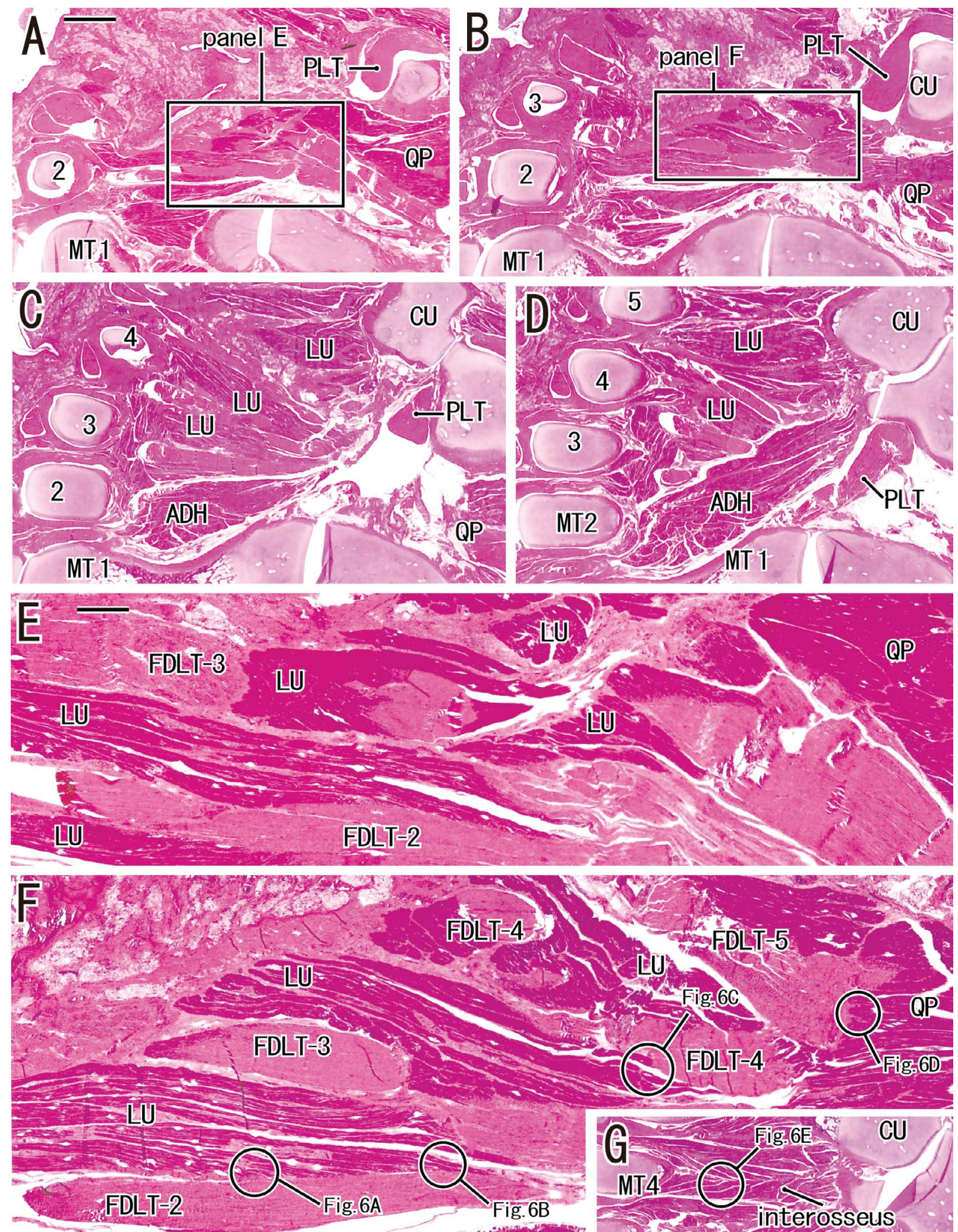

Figure 4. A-F. Foot lumbricalis origins from deep tendons near term. Crown-rump length $328 \mathrm{~mm}$. Tangential sections along the planta.

Haematoxylin and eosin staining. Panel $A$ displays the most superficial plane near the skin in the figure. Panels $E$ and $F$ are higher magnification views of squares in panels $A$ and $B$, respectively. Panel $G$, showing the interosseous muscle between the third and fourth toes, corresponds to a plane deeper than panel $F$. The lumbricalis (LU) is surrounded by the flexor digitorum longus tendons (FDLT), but due to a slightly wavy course of the tendons, the muscle origin appears to be irregularly intermingled with deep tendons (panels $E$, F). Higher magnification views of the lumbricalis origin from the deep tendon are shown in Figure 6. Panels $A-D$ (or $E$ and $F$ ) were prepared at the same magnification (scale bars: $10 \mathrm{~mm}$ in panel A; $1 \mathrm{~mm}$ in panel E); CU — cuboid; MT — metatarsal bone; PLT — peroneus longus tendon; QP — quadratus plantae. 

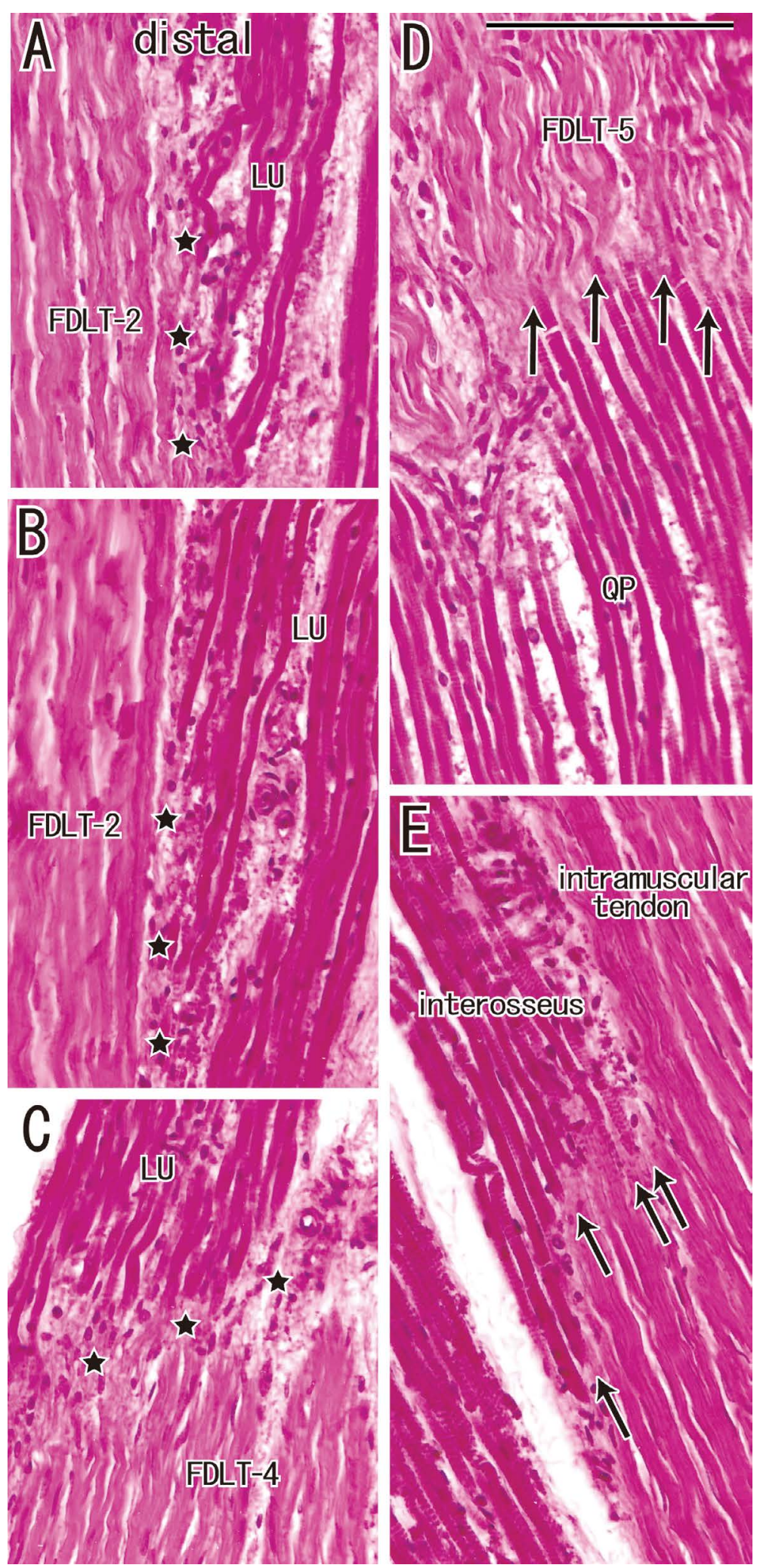

Figure 5. A-E. Histology of the lumbricalis origin: a difference from other muscle origins (higher magnification views of Fig. 4). The upper side of each panel corresponds to the distal side of the specimen. Panels A-C, corresponding to circles in Figure 4F, display foot lumbaricalis origins (LU). Panel D (corresponding to a circle in Fig. 4F) and panel E (a circle in Fig. 4G) exhibit the quadratus plantae insertion (QP) and the plantar interosseous origin, respectively. Along the flexor digitorum longus tendons (FDLT), muscle fibres of the foot lumbricalis attach to relatively loose connective tissue containing abundant tenocyte candidates (stars in panels A-C). Muscle fibres of the $\mathrm{QP}$ and interosseous are connected to a collagen fibre bundle of tendons (arrows in panels D, E). All panels were prepared at the same magnification (scale bar in panel $\mathrm{D}, 0.1 \mathrm{~mm})$. 

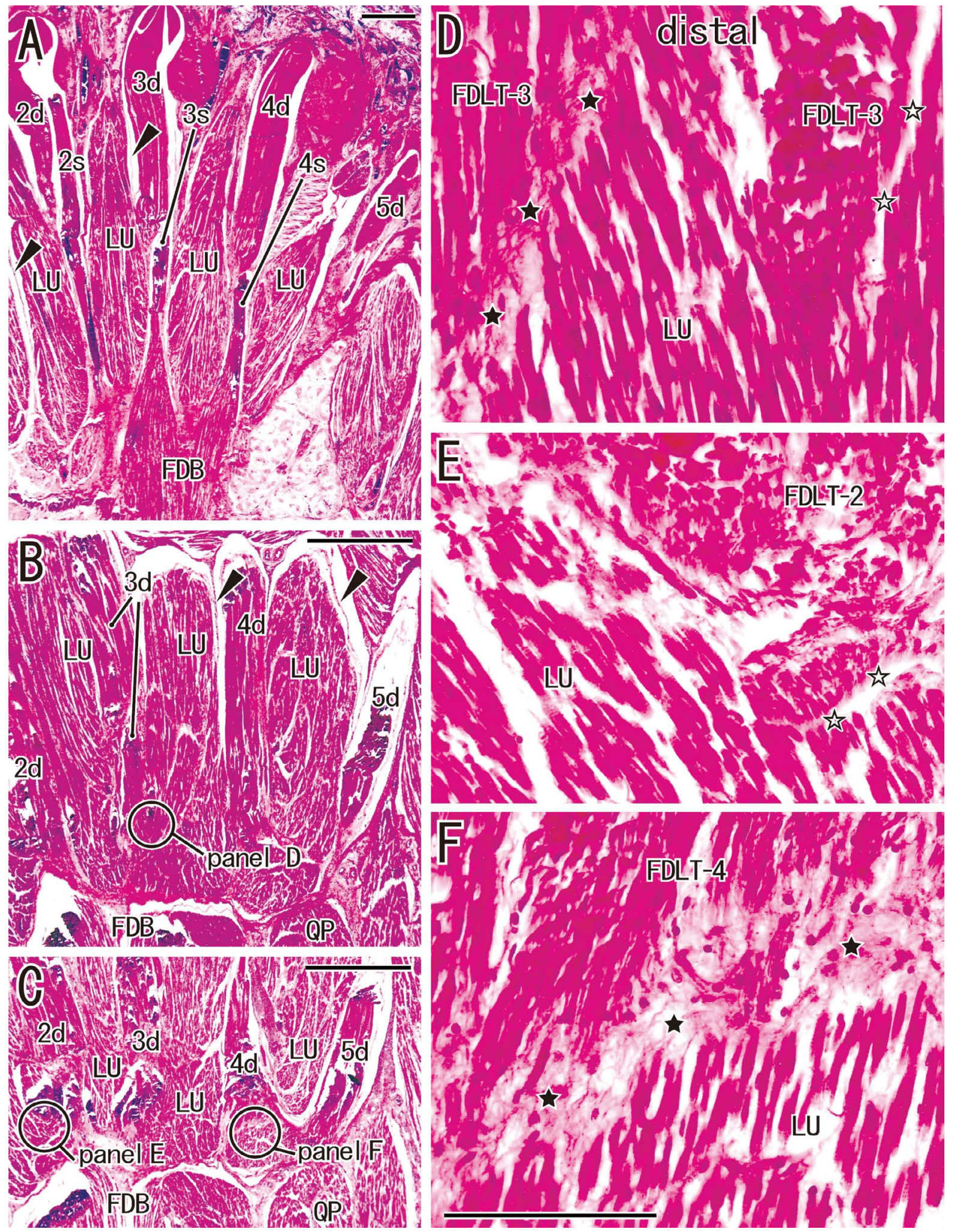

Figure 6. A-F. Increased thickness of the lumbricalis and a start of the intermingling with deep tendons. Crown-rump length $295 \mathrm{~mm}$. Tangential sections of a specimen without a direct origin of the lumbricalis (LU) from deep tendons. The proximal part of the LU is thick between superficial tendons $2 s-4 s$ in panel $A$. In deeper planes (panels B and C), the LU is surrounded by or intermingled with deep tendons $2 \mathrm{~d}-5 \mathrm{~d}$. Each of the LU muscles is surrounded by fascia (arrowheads in panels $A$ and B). Panels D-F are higher-magnification views of circles in panels $B$ and $C$. Because the flexor digitorum longus tendon (FDLT) is partly surrounded by the LU, in panels $D$ and $F$, the LU muscle fibres are seen in the proximal side of the tendon. There is fibrous tissue (fascia; black stars in panels D, F) or a narrow space (open stars in panels $D, E$ ) between the FDLT and LU. Panels D-F were prepared at the same magnification (scale bar: $1 \mathrm{~mm}$ in panels A-C; $0.1 \mathrm{~mm}$ in panel F); FDB — flexor digitorum brevis; $Q P$ — quadratus plantae. 

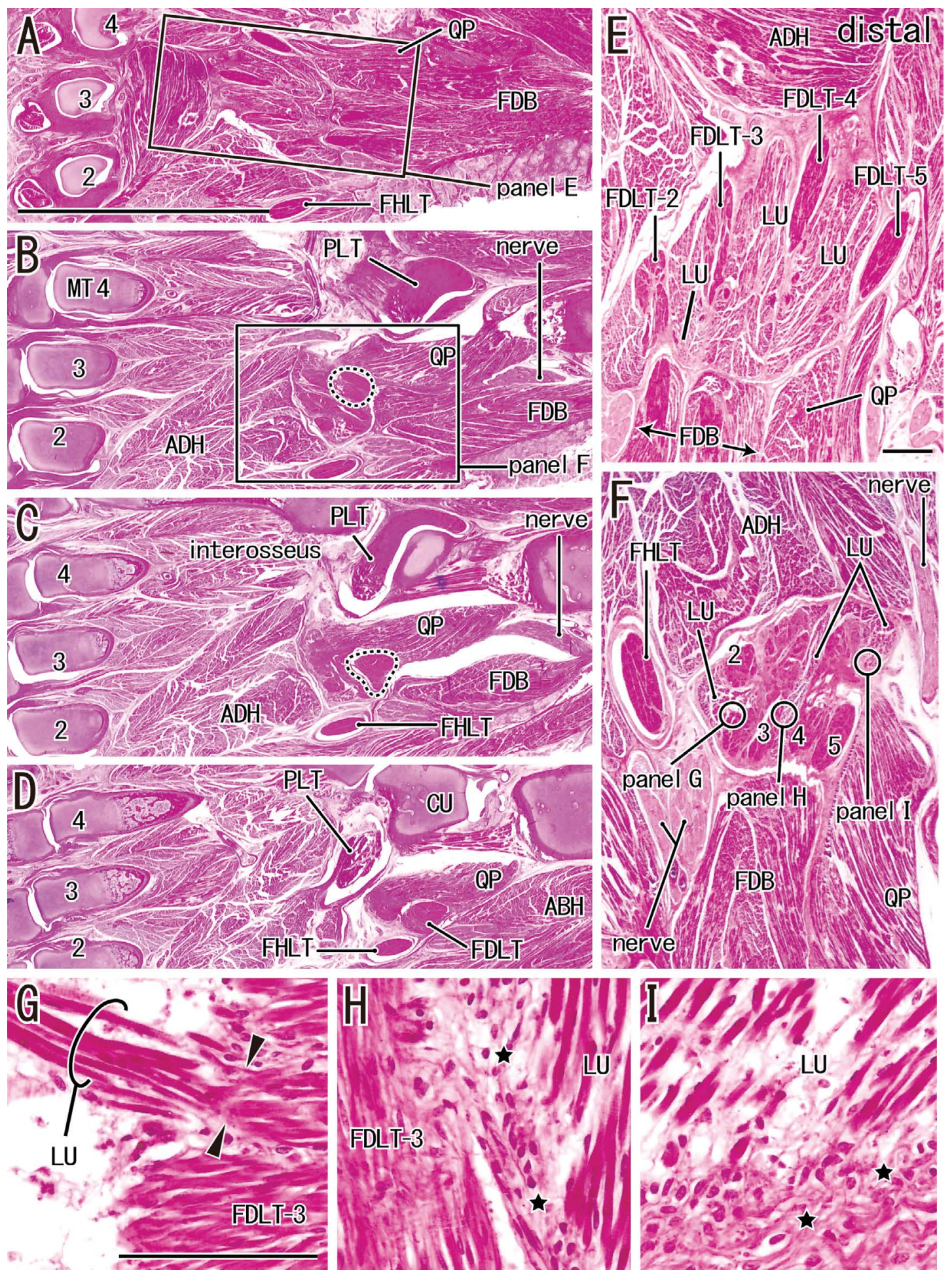

Figure 7. A-I. Intermingling between the lumbricalis and tendon near and at the division of the flexor digitorum longus near term. Crown-rump length $274 \mathrm{~mm}$. Tangential sections deeper than the flexor digitorum brevis tendons. Haematoxylin and eosin staining. Panel A displays the most superficial plane near the skin in the figure. Panels $E$ and $F$ are higher magnification views of squares in panels $A$ and $B$, respectively. Panels G-I are higher magnification views of three circles in panel F. The lumbricalis (LU) is surrounded by and intermingled with the flexor digitorum longus tendons (FDLT; panels E, F). A major part of the FDLT, encircled by a dotted line in panels $B$ and C, continues to the FDLT in panel $D$ : it is composed of the fourth and fifth deep tendons. Another part the tendons, largely from the second and third tendons, receives the quadratus plantae (QP; panels C, D). The $\mathrm{QP}$ is separated from the LU by fibrous tissue (panels E, F). Panel $\mathrm{G}$ exhibits a rare connection (arrowheads) between the LU and a deep tendon. Panels $\mathrm{H}$ and I show an interface tissue (stars) between the $L U$ and tendon. Panels $A-D$, panels $E$ and $F$ or panels $G-I$ were prepared at the same magnification, respectively (scale bar: $5 \mathrm{~mm}$ in panel $A ; 1 \mathrm{~mm}$ in panel E; $0.1 \mathrm{~mm}$ in panel G); ABH — abductor hallucis; ADH — adductor halluces; CU — cuboid; FHLT — flexor hallucis longus tendon; MT — metatarsal bone; PLT - peroneus longus tendon. 
extended superficially, as opposed to at the level of the deep tendons. Thus, in superficial sections, the LU appeared to be surrounded by the flexor digitorum brevis tendons rather than FDLT (Fig. 6A). Third, the proximal end of the LU became intermingled with collagen bundles of deep tendons at and near the dividing site of the FDLT, and they together provided a musculotendinous complex (Figs. 6C; 7). The proximal parts of the LU were divided by tendons into several clusters. Thus, some of the muscle clusters were located in the "proximal or posterior" side of the tendon. Therein, in contrast to the initial origin from the tendon, the LU distal end controversially appeared to "insert" into the FDLT (Fig. 6D-F). The intermingling and dividing process made parts of the LU surround deep tendons. Conversely, we rarely found a close relation between the muscle fibre and tendon (Fig. 7G); it was also rare to find a loose interface tissue between the $\mathrm{LU}$ and the tendon (Fig. $7 \mathrm{H}, \mathrm{I})$. In accordance with those changes, the dividing site of the FDLT appeared to migrate distally when compared with the peroneus longus tendon that transversed the foot medially in the deep side of the FDLT (Fig. 4B vs. Fig. 7B).

In the deep side of the LU, the interosseous contained a thin intramuscular tendon to provide a bipennatus appearance (Figs 4G; 7C). The quadratus plantae did not attach to the LU (Figs. 6C; 7C). In contrast to the LU origin from deep tendons, the nearby muscle origin or insertion was composed of a direct connection between a collagen fibre bundle and a muscle fibre (Fig. 5D, E). At the dividing site of the FDLT, the laterally-located elements (the fourth and fifth tendons) received the quadratus plantae, while the medially-located elements (the second and third tendons) tended to continue posteriorly to a single tendon of the flexor digitorum longus (Fig. 7A-D).

\section{DISCUSSION AND CONCLUSIONS}

The most striking observation in this study seemed to be the great variation in LU morphologies in nearterm foetuses. In contrast to a rather stable morphology in early and midterm foetuses, at and near the muscle origin near term, we found four evidences: phase 1) the LU origin was interposed by abundant cells presumed tenocytes; phase 2) the growing LU expanding the origin to the covering fascia; phase 3 ) the muscle proximal end intermingling with deep tendons to provide a musculotendinous complex; and phase 4) several proximal muscle clusters divided by ten-

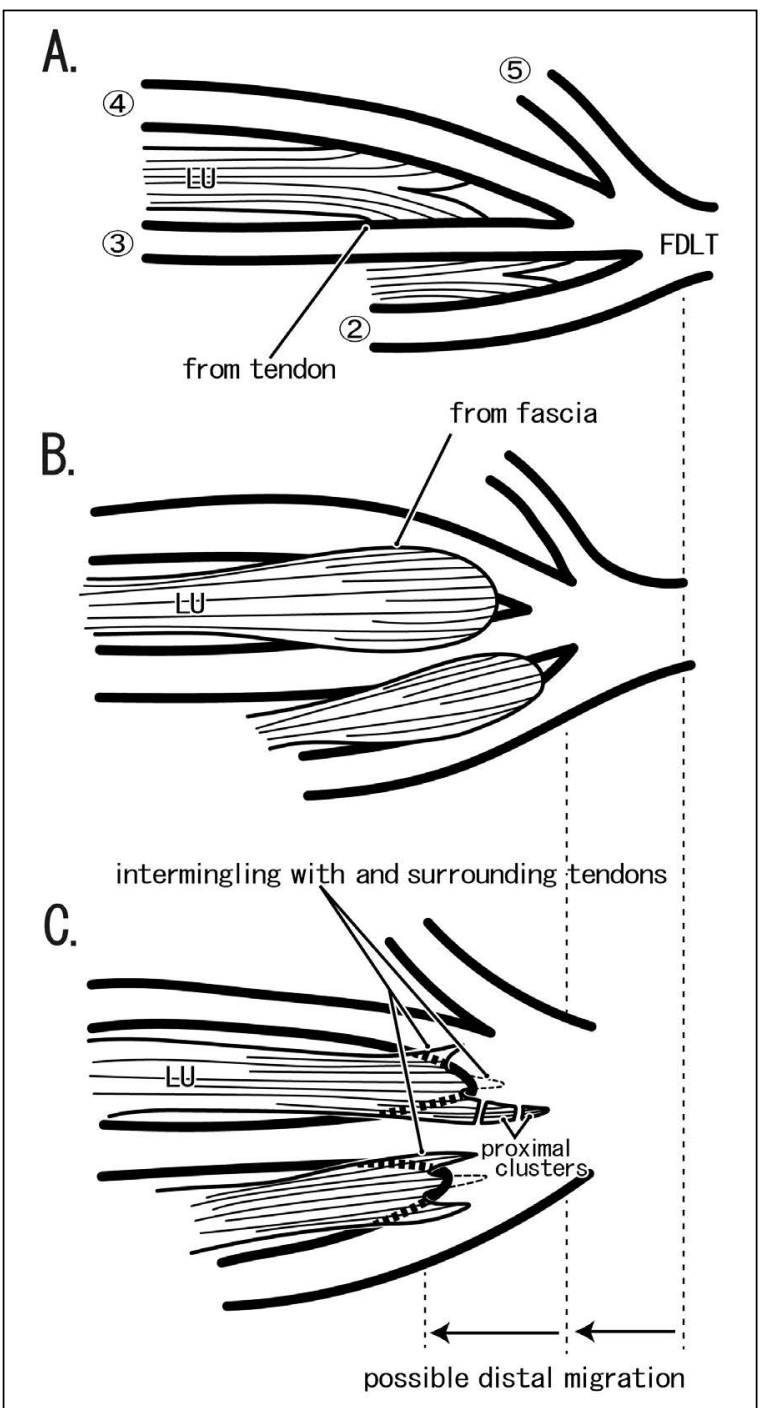

Figure 8. A-C. A hypothetical change in the lumbricalis origin from deep flexor tendons. The flexor digitorum longus tendon (FDLT) is numbered as (2), (3), (4), and (5). In the early phase (panel A), the lumbricalis (LU) originates from each division of the FDLT. In the mid-phase (panel B), the LU appears to arise from the covering fascia depending on increased thickness of the muscle. In the late phase (panel C), the LU muscle fibres intermingle with and partly surround the tendon. As these changes advance, a dividing site of the FDLT migrates distally to accelerate the involvement of the lumbricalis into tendons. The LU is not drawn between the fourth and fifth toes.

dons. These morphologies were not simple variations, but were most likely sequential changes or growth (early-, mid- and late-phases in Fig. 8). Except for phase 1, the other phases were not described in the hand, possibly because of no observations of near-term specimens [6]. Although a morphometric analysis was not performed, a distal migration of the dividing site of the FDLT was likely and it seemed to accelerate the intermingling (phase 3 ) and dividing (phase 4 ) of the proximal part of 
the LU. The distal migration of the tendon dividing site might be an analogy of the recession of flexor muscle bellies onto the foream, although the direction was reversed between the hand and foot [14]. A mixture of the $L U$ and tendon might be limited between the first and second toes, resulting in a higher incidence (around $10 \%$ ) of second LU absence in the foot $[17,18]$.

Cho et al. [6] considered that, based on observations of cross sections from midterm foetuses, the common tendinous plate is absent between the FDLT and flexor hallucis tendon. Actually, present observations also ensure the absence at midterm. However, their tangential sections were very limited in number. The present tangential sections demonstrated the foot common plate in embryos and early foetuses. Therefore, the union of all five tendons was a process common between the hand and foot. After (and independent of) programmed cell death for sculpturing the fingers/toes, the common plate seemed to appear to unite flexure tendons. A tendon to the thumb or first toe is separated from the others until midterm. However, in contrast to the hand, strict correspondence between the LU and deep tendon became lost in the near-term foot. Perhaps, the hand of near-term foetuses might also carry a morphology or variation similar to the foot LU. As shown in the quadratus plantae and interosseous, to maintain the initial muscle morphology throughout prenatal life, a definite connection seemed to be necessary between the muscle fibre and a tendon collagen bundle without an interface tissue. Leijnse [13] considered that, in the hand, tendon splitting occurs due to finger movements in utero. Limited, independent movement of the toes might make a difference in the LU between the hand and foot, although we do not have ultrasound information of living foetuses.

In the extremities, a muscle belly develops in accordance with its tendon at the same time $[4,7,15$, 20]. Foetal development of the muscle-tendon interface has been one of the leading topics in anatomical research $[1,2,22]$. In contrast to the concept of the definite connection between the muscle and tendon, our group has demonstrated a delayed morphological change at the origin or insertion of human foetus striated muscles at multiple sites $[5,9,11,12,16$, 21]. Because an anchoring of striated muscle fibre to a collagen bundle requires a large molecular complex including dystrophin, desmin, nitric oxide synthase, and other proteins, a destruction and rebuilding of the complex seemed to be unlikely in foetal development, especially in the very late stage between 30 and 40 weeks $[10,19]$. Therefore, the aforementioned delayed change in the origin and insertion seemed to occur in the "collagen side" of the enthesis, i.e., the muscle fibre attachment moves from a collagen bundle of the tendon, via that of the fascia to the musculotendinous complex at the division of the FDLP.

\section{Acknowledgements}

We are grateful to Dr. J. Männer, Institute of Anatomy in Göttingen, for his support in finding suitable specimens. This work was supported by the National Natural Science Foundation of China (No. 81460471) and the Six Talent Peaks Project of Jiangsu Province (SZCY-001).

\section{Conflict of interest: None declared}

\section{REFERENCES}

1. Abe $\mathrm{S}$, Rhee Sk, Osonoi M, et al. Expression of intermediate filaments at muscle insertions in human fetuses. J Anat. 2010; 217(2): 167-173, doi: 10.1111/j.14697580.2010.01246.x, indexed in Pubmed: 20500537.

2. Benjamin M, Ralphs JR. The cell and developmental biology of tendons and ligaments. Int Rev Cytol. 2000; 196: 85-130, doi: 10.1016/s0074-7696(00)96003-0, indexed in Pubmed: 10730214.

3. Chaplin DM, Greenlee TK. The development of human digital tendons. J Anat. 1975; 120(Pt 2): 253-274, indexed in Pubmed: 1201962.

4. Charvet B, Ruggiero F, Le Guellec D. The development of the myotendinous junction. A review. Muscles Ligaments Tendons J. 2012; 2(2): 53-63, indexed in Pubmed: 23738275.

5. Cho KH, Jin ZW, Abe $\mathrm{H}$, et al. Tensor fasciae latae muscle in human embryos and foetuses with special reference to its contribution to the development of the iliotibial tract. Folia Morphol. 2018; 77(4): 703-710, doi: 10.5603/ FM.a2018.0015, indexed in Pubmed: 29500891.

6. Cho KH, Kim JH, Ha YS, et al. Development of the deep flexor tendons and lumbricalis muscle in the hand and foot: a histological study using human mid-term foetuses. Folia Morphol. 2012; 71(3): 154-163, indexed in Pubmed: 22936550.

7. Deries M, Schweitzer R, Duxson MJ. Developmental fate of the mammalian myotome. Dev Dyn. 2010; 239(11): 2898-2910, doi: 10.1002/dvdy.22425, indexed in Pubmed: 20865781.

8. Dylevsky I. Tendons of the $\mathrm{m}$. flexor digitorum superficialis et profundus in the ontogenesis of the human hand. Folia Morphol. 1968; 16: 124-130.

9. Hayashi S, Hirouchi H, Murakami G, et al. Transient connection or origin of the inferior pharyngeal constrictor during fetal development: A study using human fetal sagittal sections. Ann Anat. 2020; 228: 151438, doi: 10.1016/j. aanat.2019.151438, indexed in Pubmed: 31726208. 
10. Heydemann A, McNally E. NO more muscle fatigue. J Clin Invest. 2009; 119(3): 448-450, doi: 10.1172/jci38618, indexed in Pubmed: 19306501.

11. Jin $Z W$, Abe $H$, Jin $Y$, et al. Positional changes in tendon insertions from bone to fascia: development of the pes anserinus and semimembranosus muscle insertion in human foetuses. Folia Morphol. 2016; 75(4): 503-511, doi: 10.5603/FM.a2016.0020, indexed in Pubmed: 27830876.

12. Jin $Z W$, Jin $Y$, Yamamoto $M$, et al. Oblique cord (chorda obliqua) of the forearm and muscle-associated fibrous tissues at and around the elbow joint: a study of human foetal specimens. Folia Morphol. 2016; 75(4): 493-502, doi: 10.5603/FM.a2016.0019, indexed in Pubmed: 27830875.

13. Leijnse JN. A generic morphological model of the anatomic variability in the $\mathrm{m}$. flexor digitorum profundus, $\mathrm{m}$. flexor pollicis longus and $\mathrm{mm}$. lumbricales complex. Acta Anat (Basel). 1997; 160(1): 62-74, doi: 10.1159/000147997, indexed in Pubmed: 9643660.

14. Lewis WH. The development of the muscular system. In: Keibel F, Mall FP (eds.). Manual of human embryology. Lippincott, Philadelphia 1910: 454-522.

15. Murchison ND, Price BA, Conner DA, et al. Regulation of tendon differentiation by scleraxis distinguishes force-transmitting tendons from muscle-anchoring tendons. Development. 2007; 134(14): 2697-2708, doi: 10.1242/dev.001933, indexed in Pubmed: 17567668.

16. Naito M, Suzuki $R$, Abe $H$, et al. Fetal development of the human obturator internus muscle with special reference to the tendon and pulley. Anat Rec (Hoboken). 2015;
298(7): 1282-1293, doi: 10.1002/ar.23121, indexed in Pubmed: 25683268.

17. Onari T. On the foot short muscles with the so-called double innervation (In Japanese with an English abstract.). Nihon Univ J Med. 1960; 19: 1411-1430.

18. Oukouchi H, Murakami T, Kikuta A. Insertions of the lumbrical and interosseous muscles in the human foot. Okajimas Folia Anat Jpn. 1992; 69(2-3): 77-83, doi: 10.2535/ ofaj1936.69.2-3_77, indexed in Pubmed: 1436952.

19. Percival JM, Anderson KNE, Huang P, et al. Golgi and sarcolemmal neuronal NOS differentially regulate contraction-induced fatigue and vasoconstriction in exercising mouse skeletal muscle. J Clin Invest. 2010; 120(3): 816-826, doi: 10.1172/JCl40736, indexed in Pubmed: 20124730.

20. Pryce BA, Watson SS, Murchison ND, et al. Recruitment and maintenance of tendon progenitors by TGF-beta signaling are essential for tendon formation. Development. 2009; 136(8): 1351-1361, doi: 10.1242/dev.027342, indexed in Pubmed: 19304887.

21. Shiraishi Y, Jin ZW, Mitomo K, et al. Foetal development of the human gluteus maximus muscle with special reference to its fascial insertion. Folia Morphol. 2018; 77(1): 144-150, doi: 10.5603/FM.a2017.0060, indexed in Pubmed: 28653302.

22. Shukunami C, Takimoto A, Nishizaki Y, et al. Scleraxis is a transcriptional activator that regulates the expression of Tenomodulin, a marker of mature tenocytes and ligamentocytes. Sci Rep. 2018; 8(1): 3155, doi: 10.1038/ s41598-018-21194-3, indexed in Pubmed: 29453333. 